\title{
On the temperature scaling behaviour of the linear magnetoresistance observed in high-temperature superconductors
}

\author{
John Singleton ${ }^{1,2}$ \\ ${ }^{1}$ National High Magnetic Field Laboratory, MS-E536, \\ Los Alamos National Laboratory, Los Alamos, NM 87545, U.S.A. \\ ${ }^{2}$ University of Oxford, Department of Physics, The Clarendon Laboratory, \\ Parks Road, Oxford, OX1 3PU, United Kingdom
}

\begin{abstract}
An analytical model invoking variations in the charge-carrier density is used to generate magnetoresistance curves that are almost indistinguishable from those produced by sophisticated numerical models. This demonstrates that, though disorder is pivotal in causing linear magnetoresistance, the form of the magnetoresistance thus generated is insensitive to details of the disorder. Taken in conjunction with the temperature $(T)$ dependence of the zero-field resistivity, realistic levels of disorder are shown to be sufficient to explain the linear magnetoresistance and field- $T$ resistance scaling observed in high-temperature pnictide and cuprate superconductors. Hence, though the $T$-linear zero-field resistance is a definite signature of the "strange metal" state of high-temperature superconductors, their linear magnetoresistance and its scaling is unlikely to be so.
\end{abstract}

In experimental tours de force ${ }^{[1]}$, the magnetoresistance of pnictide and cuprate superconductors - archetypal strange metals - has been measured in magnetic fields $\mu_{0} H$ of up to $92 \mathrm{~T}$ as a function of temperature $T$. It was found that the transverse magnetoresistance $\rho$ [measured along the length of a bar-like sample with the current $I$ applied along the bar axis, $\perp \mathbf{H}$ - see Fig. 1(a)] exhibited interesting scaling behaviour with $H$ and $T$. For instance, in Ref. 1, when $\left(\rho(H, T)-\rho_{0}\right) / T$, where $\rho_{0}$ is the residual resistivity, is plotted against $H / T$, the data map onto a single curve, which tends to a straight line (i.e. linear magnetoresistance) at larger values of $H / T$. Any observation that gives a "clue towards our... understanding of the strange metal state in high-temperature superconductors ${ }^{1}$, ' is very welcome; consequently, the linear magnetoresistance data have stimulated theoretical models such as those in Refs. 4 and 5. For example, Ref. 5 invokes a disordered strange metal consisting of itinerant electrons interacting via random couplings with naturally formed "quantum dots" containing localized electrons; the $T$-linear zero-field resistivity and some aspects of the magnetoresistance scaling are reproduced ${ }^{\sqrt{5}}$.

However, linear magnetoresistance is far from being the preserve of strange metals, having been measured in many other systems for decades (see e.g., 6-10). A particular issue was the observation of linear magnetoresistance in "simple metals" such as Al [11-13], whereas undergraduate text books ${ }^{14}$ state that this should not occur. As reviewed in Ref. 10, the necessary ingredient is disorder, the most sophisticated treatments of which are the Random Resistor Network (RRN) model and the Effective Medium Theory (EMT). The former constructs a grid of four-terminal resistors, each with a varying random resistance; the latter posits a smoothly varying disorder potential that causes a continuous variation of the local conductivity. Both models belong to the same universality class and they are in reasonable agreement with data from a diverse range of materials ${ }^{10}$.

Despite these successes, discussions at conferences in

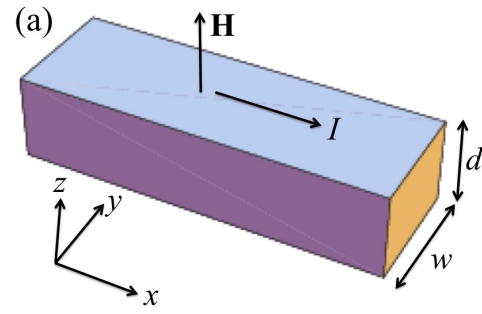

(b)

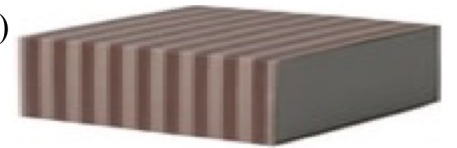

FIG. 1. (a) The geometry of the experiments modelled in this paper, carried out on bar-like samples. Arrows show the directions of the Cartesian $(x, y, z)$ axes; the point $(x, y, z)=$ $(0,0,0)$ is at the geometrical centre of the bar. The transverse magnetoresistance is obtained by measuring the voltage between two contacts placed at the same height $(z)$ on one of the faces (purple) parallel to the $x z$ plane. The current $I$ is directed along the bar $(x)$ axis, perpendicular to the applied field $\mathbf{H} \| z$. The bar is $w$ wide and $d$ thick. (b) Cartoon of a bar-like sample consisting of alternating layers that have $\kappa$ negative (brown) and positive (pink).

the past couple of years ${ }^{15}$ suggest that the RRN and EMT have little traction in the strange-metal community. The reason is perhaps that implementing these models involves the specification of inital parameters that are not straightforwardly related to the properties of real materials, plus the application of randomness, followed by a full numerical calculation; fits to data are not trivial.

Therefore, the current paper has three purposes. (i) It describes a simple, analytical model for linear magnetoresistance due to disorder characterized by a single adjustable parameter that is relatable to measurable material properties. (ii) It shows that the model reproduces the predictions of the more sophisticated RRN and EMT approaches to good accuracy, thus permitting much simpler fits of theory to data. (iii) The good fit of the model 
to data enables the $(H, T)$ scaling behaviour of the magnetoesistance of high-temperature superconductors to be explained solely in terms of the expected disorder, without the need for explicitly "strange metal" physics.

My starting point is the work of Bruls and coworkers, who showed that variations in Hall voltage $V_{\mathrm{H}}$ along the length of a bar-like sample can result in linear magnetoresistance ${ }^{11}$ 13. (For a simple metal with a spherical Fermi surface, populated by a density $n$ electrons per unit volume, the Hall voltage in a magnetic flux density $B$ is 14

$$
V_{\mathrm{H}}=-I B /(\text { ned }),
$$

where $d$ is the sample thickness and $e$ is the magnitude of the charge of the electron.) Bruls et al. proved this idea by measuring bars of very pure $\mathrm{Al}$, machined so that $d$ (and hence $V_{\mathrm{H}}$ - see Eq. 1) varied along their length 11 13. An equivalent effect is obtained by varying $n$ along the bar ${ }^{16}$; I show here that this principle can be applied to the cuprates and pnictides to generate linear magnetoresistance with the required scaling behaviour.

The first part of the derivation is similar to that in Ref. 16, however, the system dealt with in Ref. [16 is very different from the pnictides and cuprates; an exploration of linear magnetoresistance is not the primary purpose of Ref. 16, and the relevant expression is given in terms of parameters that are not easily understood 17 . Therefore I give a simplified, but complete form of the derivation (SI units, contemporary notation) in the hope that it will encourage others to play with the model and/or develop calculations from a similar starting point.

I assume a bar-like sample fabricated from a simple metal [Fig. 1(a)]; the latter has a single, negatively charged carrier type of number density $n$ and with effective mass $m^{*}$ (it is easy to show that the same result obtains for positively charged holes). If $\mathbf{H}$ is parallel to $z$ (as it is in most of the experiments ${ }^{1 / 3}$ ) it does not matter whether this material has a three-dimensional (spherical) or two-dimensional (cylindrical) Fermi surface, as long as the axis of the latter is also parallel to $z$. The current runs along $x$, parallel to the bar long axis, and the field is applied parallel to $z$. I assume that the sample is not very magnetic, so that the magnetic flux density within it and the applied field are roughly the same: i.e., $B \approx \mu_{0} H$.

The nature of the Lorentz force $(-e \mathbf{v} \times \mathbf{B})$ means that there is no change to the carrier motion in the $z$ direction ${ }^{14}$, parallel to $\mathbf{B}$. It is therefore sufficent to consider the following elements of the conductivity tensor $\underline{\sigma}$ :

$$
\begin{gathered}
\sigma_{x x}=\sigma_{y y}=\sigma^{\prime}=\frac{n e^{2} \tau}{m^{*}} \frac{1}{\left(1+\omega_{\mathrm{c}}^{2} \tau^{2}\right)} \\
\sigma_{y x}=-\sigma_{x y}=\sigma^{\prime} \omega_{\mathrm{c}} \tau .
\end{gathered}
$$

Here, $\tau$ is the scattering time within the relaxation-time approximation and $\omega_{\mathrm{c}}=\frac{e B}{m^{*}}$ is the cyclotron frequency $\sqrt{14}$.

In the steady state, the continuity equation 14 is

$$
\nabla \cdot \mathbf{j}=0
$$

where $\mathbf{j}=\underline{\sigma} \mathbf{E}$ is the current density in the sample due to electric field E. Experiments 131113 measure the voltages $V=V(x, y)$ at various points on the rod; remembering that $\mathbf{E}=-\nabla V$, Eqs. 2 and 3 combine to yield

$$
\begin{gathered}
\sigma^{\prime}\left[\frac{\partial^{2} V}{\partial x^{2}}+\frac{\partial^{2} V}{\partial y^{2}}\right]+\frac{\partial V}{\partial x}\left[\frac{\partial \sigma^{\prime}}{\partial x}+\frac{\partial\left(\omega_{\mathrm{c}} \tau \sigma^{\prime}\right)}{\partial y}\right] \\
+\frac{\partial V}{\partial y}\left[\frac{\partial \sigma^{\prime}}{\partial y}-\frac{\partial\left(\omega_{\mathrm{c}} \tau \sigma^{\prime}\right)}{\partial x}\right]=0
\end{gathered}
$$

where the possibility that $\sigma^{\prime}, \omega_{\mathrm{c}}$ and/or $\tau$ might vary with $(x, y)$ has been introduced; the disorder and inhomogeneities that might produce such variations are a common feature of many models of linear magnetoresistance in conventional metals and semiconductors 8 13]16.

The pnictides ${ }^{1 / 2} \mathrm{BaFe}_{2}\left(\mathrm{As}_{1-x} \mathrm{P}_{x}\right)_{2}$ and cuprates ${ }^{3}$ $\mathrm{La}_{2-x} \mathrm{Sr}_{x} \mathrm{CuO}_{4}$ used in the linear magnetoresistance studies are alloys, and it is inevitable that some inhomogeneity of composition (and hence $n$ ) will occur. In addition, cuprates suffer from slight variations in oxygen stoichiometry, again leading to variable $n$ (see e.g., Ref. 18 and references therein). Finally, the carrier density in both types of high-temperature superconductor is much smaller than that in conventional metals such as

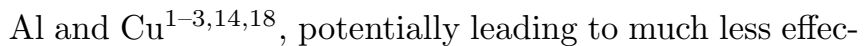
tive screening of disorder due to impurities and other defects 5 . Even in crystalline organic superconductors, relatively clean systems compared to the cuprates, this reduced screening was shown to lead to an inhomogeneous carrier density 19 . The current model therefore allows $n$ to vary with position in the sample ${ }^{\sqrt{16}}$; other quantities such as $m^{*}$ and $\tau$ are assumed to be much less affected by inhomogeneities and so are taken to be constants.

Eq. 4 could be attacked using a variety of techniques. However, my motivation is to derive a tractable expression for the magnetoresistance measured using the voltage drop in the $x$ direction. I therefore consider a variation of $n$ only in the $x$ direction (the $\sim 10 \mathrm{~nm}$ to $\sim 1 \mu \mathrm{m}$ lengthscales over which this happens will be discussed below). Separation of variables is then applied to Eq. 4 to produce an analytical solution. We hence choose ${ }^{16}$

$$
n=n_{0} \mathrm{e}^{\kappa x}
$$

with $n_{0}$ and $\kappa \neq 0$ being constants. Insertion of this into Equation 4 produces

$$
\left[\frac{\partial^{2} V}{\partial x^{2}}+\frac{\partial^{2} V}{\partial y^{2}}\right]+\kappa\left[\frac{\partial V}{\partial x}-\omega_{\mathrm{c}} \tau \frac{\partial V}{\partial y}\right]=0 .
$$

This has the solution $V=V_{0} \mathrm{e}^{-\kappa x} \mathrm{e}^{\kappa \omega_{\mathrm{c}} \tau y}+C$, where $V_{0}$ and $C$ are constants; as Refs. 1 3 deal only with voltage differences between contacts, $C$ can be set equal to 0 . (Owing to the way in which the variation of $n$ with $x$ was chosen, this solution automatically satifies the boundary condition that no current should flow out of the sample surfaces at $y= \pm \frac{w}{2}$ [Fig. 11(a)].) 

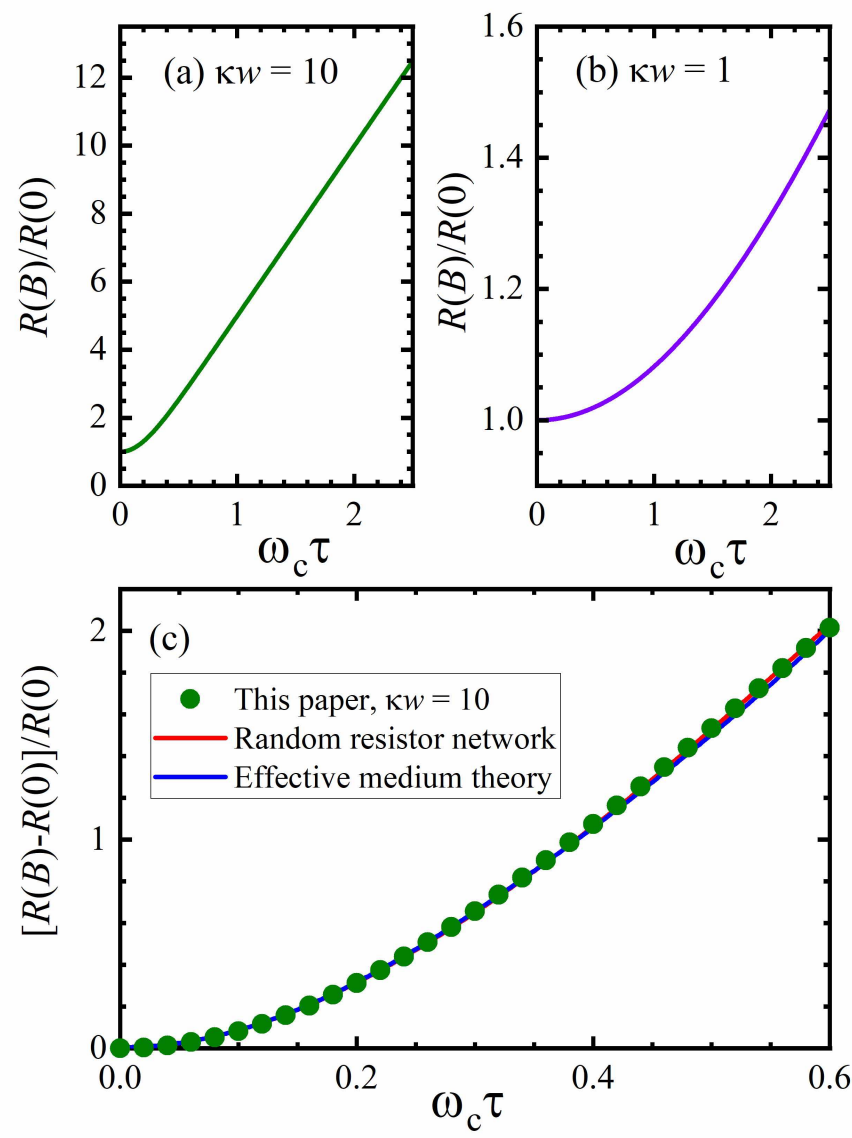

FIG. 2. (a,b) Eq. 11 plotted against $\omega_{\mathrm{c}} \tau$ for $|\kappa w|=10[(\mathrm{a})$, green curve] and $|\kappa w|=1$ [(b), purple curve]. (c) Comparison of part of the curve from (a) [green points] with the predictions of the Random Resistor Network model [RRN, red curve] and the Effective Medium Theory [EMT, blue curve 10 . Note that the EMT curve covers the RRN curve for most of the figure. Above $\omega_{\mathrm{c}} \tau \approx 0.3$, the results of all three models lie on straight lines [i.e., all predict linear magnetoresistance] to good degree of accuracy.

The fact that a current $I$ flows along the sample bar (Figure 1) is used to find $V_{0}$. We have

$$
\begin{gathered}
I=d \int_{-\frac{w}{2}}^{+\frac{w}{2}} j_{x} \mathrm{~d} y \\
=\sigma^{\prime} \kappa V_{0} \mathrm{e}^{-\kappa x}\left(1+\omega_{c}^{2} \tau^{2}\right) d \int_{-\frac{w}{2}}^{+\frac{w}{2}} \mathrm{e}^{\kappa \omega_{c} \tau y} \mathrm{~d} y ;
\end{gathered}
$$

some rearrangement gives $V_{0}$, yielding

$$
V=\frac{I}{\sigma_{0}} \frac{1}{\kappa d w}\left[\frac{\frac{\kappa w \omega_{\mathrm{c}} \tau}{2}}{\sinh \left(\frac{\kappa w \omega_{\mathrm{c}} \tau}{2}\right)}\right] \mathrm{e}^{-\kappa x} \mathrm{e}^{\kappa \omega_{\mathrm{c}} \tau y},
$$

where $\sigma_{0}=n_{0} e^{2} \tau / m^{*}$. Note that the central bracket tends to the value 1 in a well-behaved fashion as $\omega_{\mathrm{c}} \rightarrow 0$.

In order to understand the data in Refs. 1 3 , consider voltages $V_{1}$ and $V_{2}$ at two contacts on the same face $[\perp y$; Figure [1(a)] of the bar at positions $(x, y)=\left(x_{1}, \frac{w}{2}\right)$ and $\left(x_{2}, \frac{w}{2}\right)$ respectively. Evaluating the voltage difference
$\Delta V(B)=V_{1}-V_{2}$ at both $B=0$ and finite $B$, and combining these two expressions gives

$$
\Delta V(B)=\Delta V(0) \frac{\kappa w \omega_{\mathrm{c}} \tau}{1-\mathrm{e}^{-\kappa w \omega_{\mathrm{c}} \tau}}
$$

or, in terms of the measured resistance, $R(B)=$ $\Delta V(B) / I$,

$$
R(B)=R(0) \frac{\kappa w \omega_{\mathrm{c}} \tau}{1-\mathrm{e}^{-\kappa w \omega_{\mathrm{c}} \tau}}, \quad \text { with } \quad \omega_{\mathrm{c}}=\frac{e B}{m^{*}} .
$$

For $\omega_{\mathrm{c}} \tau>0$, the exponential in the denominator of Eq. 10 decreases with increasing $B$, leading a dependence dominated by $\omega_{\mathrm{c}} \tau$ in the numerator; the magnetoresistance thus becomes positive and linear. For $\omega_{\mathrm{c}} \tau<0$, the exponental grows, leading to negative magnetoresistance.

The reason that Eq. 10 is asymmetric about $B=0$ is that I have used $\kappa>0$, breaking the longitudinal symmetry of the bar. However, unless the sample has been crafted with an alloy composition that changes along the length of the bar ${ }^{3 / 16}$, one would expect $n(x)$ to vary both up and down about some average value. A minimalist approach is to imagine that the rod consists of a stack of slabs along the $x$ direction in which $\kappa$ alternates between negative and positive [Fig. 1(b)]. For a large number of slabs the resistance will be the average of Equation 10 evaluated for $\kappa>0$ and $\kappa<0$ :

$$
R(B)=R(0) \frac{\kappa w \omega_{\mathrm{c}} \tau}{2}\left[\frac{1}{1-\mathrm{e}^{-\kappa w \omega_{\mathrm{c}} \tau}}-\frac{1}{1-\mathrm{e}^{\kappa w \omega_{\mathrm{c}} \tau}}\right] .
$$

This function, now symmetrical about $B=0$, is all that is needed for an understanding of the data in Refs. 13, it is plotted in Figs. 2(a,b). Note that the magnetoresistance is a function of only the zero-field resistance and the product of two dimensionless quantities, $\omega_{\mathrm{c}} \tau$ and $\kappa w$; it will therefore follow exactly the same temperature dependence as $R(0)$ does. The shape is determined by the dimensionless quantities; increasing $\kappa w$ moves the crossover from curved to linear magnetoresistance to lower values of $\omega_{\mathrm{c}} \tau$ [compare Figs. 2(a) and (b)].

Fig. 2(c) compares the lower part of the model curve shown in (a) [Eq. 11 with $|\kappa w|=10$ ] with numerical results from the RRN and EMT approaches. The RRN and EMT calculations employed the parameters used to generate the curves shown in Fig. 2 of Ref. 10; the numerical scaling routine described in that paper ${ }^{20}$ was used to match the $\omega_{\mathrm{c}} \tau$ range of the various models, chosen to show the transition from curved to linear magnetoresistance in detail. There is very little difference between the form of Eq. 11 and the results of the more sophisticated numerical approaches 10 ; above $\omega_{\mathrm{c}} \tau \approx 0.3$, the points from all three models lie quite accurately on very similar straight lines. In the rough and tumble of fitting experimental data, such small differences will hardly matter. In the prediction of linear magnetoresistance, it seems that whilst disorder is of paramount importance, the fine details of the disorder matter little.21

The model curve in Fig. 2(a) is already very similar to data measured in Ref. 1 [see Fig. 3]. It therefore remains to explain the scaling behaviour. Both pnictide 


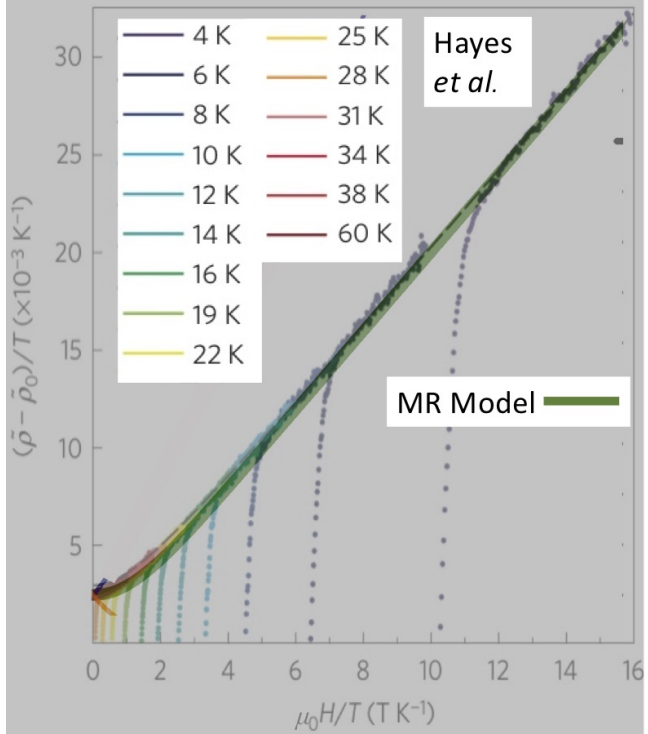

FIG. 3. Figure from Ref. 1 showing magnetoresistance data plotted as $\left(\rho-\rho_{0}\right) / T$ versus $\mu_{0} H / T$; as discussed in the text, the latter quantity is proportional to $\omega_{\mathrm{c}} \tau$. The green curve is Eq. 11 employing (see text) $|\kappa w|=10$, with $\omega_{\mathrm{c}} \tau \approx 2.5$ at $\mu_{0} H \approx 90 \mathrm{~T}$ [the upper limit of the $x$-axis].

and cuprate superconductors have a normal-state, zerofield resistance that is written as $\sqrt{1}[3$

$$
\rho(0, T)=\rho_{0}+A T
$$

where $\rho_{0}$ is a residual resistivity, assumed to be unaffected $^{[22}$ by $H$ or $T$ in Refs. 1 and 2 . It is customary to subtract $\rho_{0}$ from the data and treat the $H$ - and $T$-dependence of what remains; I do the same. For a fixed value of $\kappa w$, Eq. 11 takes the form $R(B, T)=$ $R(0, T) f\left(\omega_{\mathrm{c}} \tau\right)$, i.e. the zero-field resistance multiplied by a function of only $\omega_{\mathrm{c}} \tau$. If $R(0, T)=A T$, then

$$
R(B, T)=A T f\left(\omega_{\mathrm{c}} \tau\right) .
$$

Therefore, the magnetoresistance scales linearly with $T$, as observed ${ }^{1 / 3}$. Moreover, if one plots $R(B, T) / T=$ $A f\left(\omega_{\mathrm{c}} \tau\right)$, the data should all collapse onto a single curve that is a function of only $\omega_{\mathrm{c}} \tau$.

In the absence of temperature-dependent variations in carrier density (not expected in a metallic system ${ }^{14}$ ), the resistance will be proportional to a $T$-dependent scattering rate. Put simply, $R(0, T) \propto 1 / \tau$; since $R(0, T)=A T$, this implies that $\tau \propto 1 / T$. Therefore, if $B$ is divided by $T$, one obtains a quantity proportional to $\omega_{\mathrm{c}} \tau$. This is likely to be the reason why the magnetoresistance data from Refs. 13 divided by $T$ collapse onto a single curve when plotted versus $B / T$ [e.g., Fig. 3 .

Having given a plausible explanation for the scaling behaviour in Refs. 1 1 3, it remains to discuss reasonable values for the dimensionless quantities in the model that can be used to fit such data.

Values of $\kappa w$ : Based on general properties of fairly disordered alloys 23 , one might expect variations of $n$ of a few tenths of a percent to a few percent over the lengthscale of typical microstructure, which can range in size from $\sim$ $10 \mathrm{~nm}$ to $\sim 1 \mu \mathrm{m}$. Therefore, $\kappa$ may be $\sim 10^{4}-10^{6} \mathrm{~m}^{-1}$. Typical samples used for pulsed-field transport measurements tend to have a width $w \approx 50-500 \mu \mathrm{m}$ (author's observation). Taking values somewhere in the middle of these ranges, one obtains $\kappa w \sim 10$, as used in Fig. 2(a).

Values of $\omega_{\mathrm{c}} \tau$ in the cuprates and pnictides: Even goodquality cuprate superconductors that exhibit Shubnikovde Haas and de Haas-van Alphen oscillations in high fields have relatively large scattering rates; a typical sample of this sort ${ }^{18}$ has $\omega_{\mathrm{c}} \tau \approx 1$ at $20 \mathrm{~T}$. The samples used in Refs. 1-3 do not exhibit quantum oscillations; they are chosen to be close to the quantum-critical point and possess heavy masses and enhanced scattering. I have guessed $\omega_{\mathrm{c}} \tau \approx 2.5$ at $\mu_{0} H \approx 90 \mathrm{~T}$ [the upper limit of the $x$-axis of Figure 3. However, note that lower values of $\omega_{\mathrm{c}} \tau$ can be compensated for by higher values of $\kappa w$, still well within the plausible limits discussed above.

Inserting these estimates of $\omega_{\mathrm{c}} \tau$ and $\kappa w$ into Eq. 11 produces the green trace overlaying the data in Fig. 3 . The model matches the scaling behaviour of the experiments well, including the curvature seen at low $H$.

One further consequence of high scattering rates is that the fine details of the Fermi-surface topology are unlikely to be important in determining the transport properties; hence a model that assumes a simple Fermi-surface toplogy, such as the one presented here, may be adequate to derive general qualitative features of the resistivity.

Finally, I return to Ref. 5, which models a diffusive marginal Fermi liquid (MFL) to obtain a resistivity proportional to $T$. It is notable that the MFL alone does not give linear magnetoresistance ${ }^{5}$; only when a macroscopically disordered sample with domains of MFLs with varying densities of electrons is introduced via EMT does the linear magnetoresistance with the correct $(H, T)$ scaling appear ${ }^{5}$. This again supports the idea that the linear magnetoresistance is not caused by "strange metal" physics; rather, it is merely a consequence of disorder.

In summary, an analytical model invoking realisticsized variations in the charge-carrier density is used to generate magnetoresistance curves that are almost indistinguishable from those produced by the more sophisticated RRN and EMT models. This demonstrates that, though disorder is pivotal in causing linear magnetoresistance, the form of the magnetoresistance thus generated is rather insensitive to the microscopic details of the disorder ${ }^{10}$. Hence, the analytical expression derived [Eq. 11$]$ can be used with confidence to fit the linear magnetoresistance and $(H, T)$ scaling reported in Refs. 1-3. The good correspondence between model and data, using plausible values for the two fit parameters, shows that disorder of the size expected in alloys is sufficent to explain the linear magnetoresistance and the way in which it scales with the $T$-linear zero-field resistivity that is a feature of pnictides and cuprates 1 . Therefore, though the latter $T$-linear zero-field resistance is indeed a signature of the "strange metal" state of high-temperature superconductors ${ }^{5}$, the linear magnetoresistance need not be. 


\section{ACKNOWLEDGMENTS}

This work was supported by the US DoE Basic Energy Science Field Work Project Science in 100 T, and carried out at the National High Magnetic Field Laboratory, which is funded by NSF Cooperative Agreement DMR1157490 and 1164477, the State of Florida and U.S. DoE.
I thank the University of Oxford for the provision of a Visiting Professorship that permitted calculations that underpin this paper. Paul Goddard, Stephen Blundell, Andrea Schmidt, Arkady Shekhter, Nikola Maksimovich and Neil Harrison are thanked for valuable discussions.I am grateful to Ross McDonald for allowing me to reproduce the results from Ref. 1 plotted in Fig. 3
${ }^{1}$ Ian M. Hayes, Ross D. McDonald, Nicholas P. Breznay, Toni Helm, Philip J. W. Moll, Mark Wartenbe, Arkady Shekhter and James G. Analytis, Nature Physics, 12, 916 (2016).

2 Ian M. Hayes, Zeyu Hao, Nikola Maksimovic, Sylvia K. Lewin, Mun K. Chan, Ross D. McDonald, B. J. Ramshaw, Joel E. Moore, James G. Analytis, Phys. Rev. Lett. 121, 197002 (2018).

${ }^{3}$ P. Giraldo-Gallo, J. A. Galvis, Z. Stegen, K. A. Modic, F. F. Balakirev, J. B. Betts, X. Lian, C. Moir, S. C. Riggs, J. Wu, A. T. Bollinger, X. He, I. Bozovic, B. J. Ramshaw, R. D. McDonald, G. S. Boebinger, A. Shekhter, Science, 361 479 (2018).

${ }^{4}$ S. Cremonini, A. Hoover, and L. Li, J. High Energ. Phys. 2017, 133 (2017).

5 A.A. Patel, J. McGreevy, D.P. Arovas and S. Sachdev, Phys. Rev. X 8, 021049 (2018).

6 A.A. Abrikosov, Europhysics Letters, 49, 789 (200).

7 A.A. Abrikosov, J. Phys. A: Math. Gen. 369119 (2003).

8 M.M. Parish and P.B. Littlewood, Nature 426, 162 (2003).

9 D. Stroud, Phys. Rev. B 12, 3368 (1975).

10 Navneeth Ramakrishnan, Ying Tong Lai, Silvia Lara, Meera M. Parish, and Shaffique Adam, Phys. Rev. B 96 224203 (2017)

11 G.J.C.L. Bruls, J. Bass, A.P. van Gelder, H. van Kempen and P. Wyder, Phys. Rev. Lett. 46, 553 (1981).

12 G.J.C.L. Bruls, J. Bass, A.P. van Gelder, H. van Kempen and P. Wyder, Phys. Rev. B 32, 1927 (1985)

13 G.J.C.L. Bruls, Ph. D. Thesis, Katholieke Universiteit Nijmegen (Krips Repro, Meppel, 1985).

14 See e.g., Neil W. Ashcroft and N. David Mermin, Solid State Physics (Holt, Rinehart and Winston, 1976) or J. Singleton Band theory and electronic properties of solids (Oxford University Press, Oxford, 2002), Chs. 1 and 10.

15 For example, satellites of the International Conference on Strongly Correlated Electron Systems 2019 (SCES '19) and the American Physical Society March Meeting 2019.

16 R.T. Bate and A.C. Beer, J. Appl. Phys. 32, 800 (1961).

17 So much so that the current author was forced to rederive all of the expressions to check that he understood the various ill-defined $\rho$ parameters.

18 B. J. Ramshaw, Baptiste Vignolle, James Day, Ruixing Liang, W. N. Hardy, Cyril Proust and D. A. Bonn, Nature
Physics, 7, 234 (2011).

19 J. Singleton, N. Harrison, C.H. Mielke, J.A. Schlueter and A.M. Kini, J. Phys.: Condens. Matter, 13, L899 (2001).

${ }^{20}$ In Fig. 2 of Ref. 10, magnetoresistance curves with disorder parameters $\eta=0.1$ and $\eta=10$ from both the RRN and EMT models are indistinguishable to the naked eye, so $\eta=1$ is used here. The normalization routine of Ref. 10 looks for the point at which the low- $B$ part of the magnetoresistance deviates from a parabola and uses this for field-axis scaling.

${ }^{21}$ Similarly, in a real sample, the composition variations will be more random than the simple case described in this paper. One could deal with this by evaluating Eq. 11 for many different values of $\kappa$ and averaging the results. However, a few quick calculations (left as an exercise for the reader) will show that this does very little to change the form of the magnetoresistance.

22 The fact that the resistivity is treated in Refs. 1-3 as the sum of two completely independent terms suggests that samples look like a series-connected network of "dead" regions (giving the residual resistivity, $\rho_{0}$ ), and "alive" regions that possess quasiparticles that respond to field and temperature. This is part of the reasoning behind treatments such as that of Ref. 5 .

23 See e.g., Fundamentals of Metallurgy (1st Edition), Ed. S Seetharaman (Woodhead Publishing, Cambridge, 2005).

24 In some cases, experiments are carried out using contacts made using wires connected to a smear of silver paint or silver epoxy across the whole width of the top surface $(\perp z$ see Fig. 1) of a bar (author's observation). Such contacts frequently have a resistance $\sim 10-100$ times the fourcontact resistance of the bar and hence do not represent an equipotential region. Instead, they will detect an average of the voltages from different $y$ values across the bar, meaning that the form of the magnetoresistance will be essentially the same as Eq. 11 Note that, because the current model considers only one-dimensional variations in $n$, it cannot be used to predict quantitative differences between different contact geometries (e.g., computing the relative magnitudes of voltages from pairs of contacts on the bar faces perpendicular to $z$ and to $y$ ). 